\title{
VEGF expression and microvascular responses to severe transient hypoxia in the fetal sheep brain
}

\author{
Ana A. Baburamani ${ }^{1,2}$, Margie Castillo-Melendez ${ }^{1}$ and David W. Walker ${ }^{1,3}$
}

BACKGROUND: Fetal hypoxia contributes significantly to the pathogenesis of permanent perinatal brain injury. We hypothesized that hypoxia-induced cerebral angiogenesis and microvascular changes would occur in fetal sheep subjected to a severe hypoxic insult produced by umbilical cord occlusion (UCO) for $10 \mathrm{~min}$.

METHODS: At 124-126 d of gestation, singleton fetal sheep underwent surgery for implantation of catheters and placement of an inflatable cuff around the umbilical cord. A 10-min UCO or sham UCO $(n=5)$ was induced at $130 \mathrm{~d}$ gestation. The fetal brain was collected at $24 \mathrm{~h}(n=5)$ or $48 \mathrm{~h}(n=4)$ after UCO for immunohistochemical analysis of vascular endothelial growth factor (VEGF), Ki67, and serum albumin.

RESULTS: By 48h after UCO, the percentage of blood vessels expressing VEGF had increased in the subventricular zone, periventricular and subcortical white matter, corpus callosum, and cortex. Alterations in vascular permeability (albumin extravasation) were observed only in the periventricular and subcortical white matter and the subventricular zone following UCO.

CONCLUSION: The upregulation of VEGF expression and increased leakage of plasma protein in the fetal sheep brain show that the microvasculature in white matter is sensitive to hypoxia in the near-term brain.

C inical studies have established that hypoxic-ischemic encephalopathy can arise before birth (1) and not only injure neurons and white matter $(2,3)$ but also cause cerebral edema and subcortical hemorrhage (4). Involvement of the cerebral vasculature in events leading to brain injury is also suggested by the findings of Aly et al. (5), who found increased vascular endothelial growth factor (VEGF) protein in cord blood of fullterm human infants who had experienced perinatal asphyxia, many of whom then developed hypoxic-ischemic encephalopathy. A strong positive correlation also exists for VEGF concentrations in the placenta and neonatal cerebrospinal fluid and the severity of hypoxic-ischemic encephalopathy $(6,7)$. Increased VEGF expression in neovascular sprouts around necrotic foci in the brain of infants with periventricular leukomalacia (8) further supports the close relationship of increased VEGF and hypoxic-ischemic encephalopathy in term infants.
VEGF is expressed by vascular components such as endothelial cells and pericytes in the human fetal brain $(8,9)$, and studies in the rat suggest that neuronal expression of VEGF provides a signal for angiogenesis; furthermore, expression of VEGF in the "end-feet" of astrocytes coincides with their investment around blood vessels, an essential step in the formation of a functionally tight blood-brain barrier (10). Hypoxia induces robust upregulation of a number of oxygen-sensitive genes, including hypoxia inducible factor-1 $\alpha$ and $\operatorname{VEGF}(11,12)$. Increased VEGF expression can induce angiogenesis, and although this may eventually result in increased vascularization of ischemic tissue (13-15), newly formed blood vessels are prone to leakage and mechanical disruption $(16,17)$, as shown for tumor growth (18), wound healing (19), and adult stroke $(15,20)$. However, the effect of hypoxia-ischemia on VEGF expression in the fetal and neonatal brain, particularly associated with small blood vessels, is less well understood.

Rodent studies of neonatal hypoxia report elevated VEGF in the brain $(11,12,14)$ but have not specifically assessed vascular-associated VEGF expression; nor do these studies allow assessment of the changes that may occur in the fetal brain in utero. In the current study, we produced global systemic hypoxia by umbilical cord occlusion (UCO) in fetal sheep at $130 \mathrm{~d}$ of gestational age ( 0.9 gestation). Fetal sheep at this stage have a comparable brain development to the human fetus of $\sim 37-42 \mathrm{wk}$ (21). Therefore, this model of in utero cord compromise allows for a better investigation into the possible mechanisms that may contribute to term brain injury following an asphyxic insult, given that it produces predominantly neuronal injury in the hippocampus $(3,22,23)$.

We hypothesized that a single, severe hypoxic event would induce upregulation of VEGF associated with small blood vessels, and furthermore, this would be associated with endothelial cell proliferation, increased incidence of vascular permeability, and leakage of plasma proteins into the parenchyma of the brain. In particular, we sought to establish if the effect of this hypoxic insult was different between the gray and white matter regions of the developing brain, which might explain the greater propensity for necrosis and cystic lesions to occur in white matter regions. 


\section{RESULTS}

Successful interruption of umbilical blood flow resulted in significant hypoxia, hypercapnia, and acidemia in all fetuses subjected to UCO, as shown in Table 1, and as previously reported $(24,25)$. UCO resulted in an initial abrupt increase in fetal arterial pressure and bradycardia (see Table 2), but after $\sim 2 \mathrm{~min}$, fetal arterial pressure slowly began to fall and the heart rate increased. On release of the cuff, both arterial pressure and heart rate increased significantly over $15-30 \mathrm{~min}$, and by $1 \mathrm{~h}$ after UCO, fetal arterial pressure was near the normal range, although heart rate remained higher than the pre-UCO heart rate, and higher than for the sham UCO fetuses (Table 2). Arterial blood gases $\left(\mathrm{pO}_{2}\right.$ and $\left.\mathrm{pCO}_{2}\right)$ had recovered to be no different from pre-UCO or sham UCO values by $1 \mathrm{~h}$ after UCO, although the UCO fetuses remained slightly acidemic at this time (Table 2). All fetuses survived the cord occlusion and appeared to be physiologically normal at autopsy at either 24 or $48 \mathrm{~h}$ after UCO.

VEGF was widely expressed in the control (sham UCO) fetal sheep brain, with strong colocalization with microtubule-associated protein 2-positive neurons in the cortex and stria terminalis, with low colocalization with glial fibrillary acidic protein-positive cells in the brain regions examined. No changes in expression were seen following UCO. An

Table 1. Fetal blood gas parameters and $\mathrm{pH}$ before, during, and after UCO or sham UCO

\begin{tabular}{lccc}
\hline & Pre-UCO $(-5 \mathrm{~min})$ & $\mathrm{UCO}(9 \mathrm{~min})$ & Post-UCO $(1 \mathrm{~h})$ \\
\hline $\begin{array}{l}\mathrm{pO}_{2}(\mathrm{~mm} \mathrm{Hg}) \\
\text { Control }\end{array}$ & $21.14 \pm 0.97$ & $21.42 \pm 0.81$ & $21.30 \pm 0.71$ \\
$\mathrm{UCO}$ & $23.59 \pm 0.95$ & $12.04 \pm 0.70^{+}$ & $25.99 \pm 0.81^{* *}$ \\
$\mathrm{pCO}_{2}(\mathrm{~mm} \mathrm{Hg})$ & & & \\
$\mathrm{Control}$ & $45.60 \pm 1.71$ & $45.18 \pm 1.71$ & $45.16 \pm 1.79$ \\
$\mathrm{UCO}$ & $45.27 \pm 1.51$ & $105.92 \pm 4.95^{+}$ & $48.20 \pm 1.05$ \\
$\mathrm{pH}$ & & & \\
$\mathrm{Control}$ & $7.37 \pm 0.01$ & $7.36 \pm 0.01$ & $7.37 \pm 0.01$ \\
UCO & $7.38 \pm 0.01$ & $6.97 \pm 0.03^{+}$ & $7.29 \pm 0.03^{*}$ \\
\hline
\end{tabular}

Values for $\mathrm{pO}_{2^{\prime}} \mathrm{pCO}_{2^{\prime}}$ and $\mathrm{pH}$ from control $(n=5)$ and $\mathrm{UCO}(n=9)$ fetal sheep. Values expressed as mean \pm SEM.

UCO, umbilical cord occlusion

${ }^{*} P<0.05,{ }^{* *} P<0.01,{ }^{\dagger} P<0.001$, as compared with control. example of VEGF immunoreactivity for the corpus callosum is shown in Figure 1. With respect to specific VEGF-associated labeling of blood vessels, this was generally lower in white matter than in gray matter, with the highest proportion of VEGFpositive blood vessels occurring in the thalamus and the lowest in the subcortical white matter (Table 3). The UCO resulted in a significant increase in the relative number (\%) of blood vessels associated with expression of VEGF in the subventricular zone and the periventricular and subcortical white matter at both 24 and $48 \mathrm{~h}$ after UCO, and in the corpus callosum at $48 \mathrm{~h}$ after UCO (Table 3). In addition to the cortex (examined at the level of the motor and somatosensory cortexes), in which blood vessel-associated VEGF expression was increased at both postmortem times, the only other neuron-rich area in the brain in which vascular-associated VEGF expression was increased was the medial geniculate nucleus of the thalamus at $48 \mathrm{~h}$ post-UCO (Table 3 ).

In contrast to VEGF labeling of blood vessels, UCO did not significantly change the number of VEGF-positive cells in most brain regions (e.g., corpus callosum, cortex, subventricular zone, stria terminalus, thalamus, and periventricular and subcortical white matter) at either 24 or $48 \mathrm{~h}$ after the cord

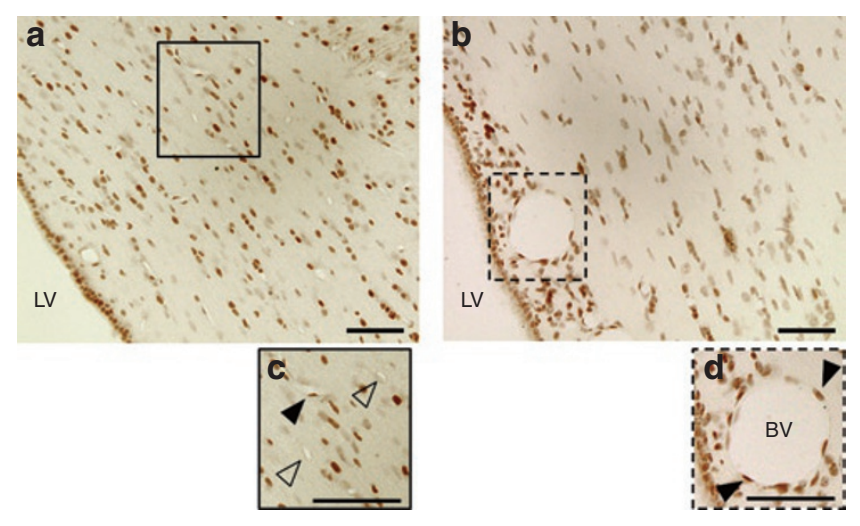

Figure 1. VEGF immunohistochemistry in fetal sheep brain. Photomicrographs show VEGF immunohistochemistry in the corpus callosum from $(\mathbf{a}, \mathbf{c})$ control and $(\mathbf{b}, \mathbf{d}) 48 \mathrm{~h}$ post-UCO. $(\mathbf{c}, \mathbf{d})$ Filled arrowheads indicate VEGF-positive staining associated with blood vessel, and (c) open arrowheads indicate no positive staining associated with blood vessel. Taken at $\times 400$ original magnification. Bar $=50 \mu \mathrm{m}$. BV, blood vessel; LV, lateral ventricle; UCO, umbilical cord occlusion; VEGF, vascular endothelial growth factor.

Table 2. Fetal mean arterial blood pressure and heart rate before, during and after UCO or sham UCO

\begin{tabular}{|c|c|c|c|c|c|}
\hline & Pre-UCO (-5 min) & UCO ( 2 min) & UCO (9 min) & Post-UCO (15 min) & Post-UCO (60 min) \\
\hline \multicolumn{6}{|c|}{ MAP (mm Hg) } \\
\hline Control & $37.90 \pm 1.65$ & $35.16 \pm 1.90$ & $34.27 \pm 1.40$ & $35.97 \pm 1.39$ & $35.00 \pm 2.28$ \\
\hline \multicolumn{6}{|c|}{ HR (beats/min) } \\
\hline Control & $160.29 \pm 8.53$ & $150.91 \pm 2.85$ & $149.58 \pm 3.27$ & $150.33 \pm 2.30$ & $151.22 \pm 9.56$ \\
\hline
\end{tabular}

Values for MAP and HR from control $(n=5)$, UCO $(n=9)$ fetal sheep. Values expressed as mean \pm SEM.

$\mathrm{HR}$, heart rate; MAP, mean arterial blood pressure; UCO, umbilical cord occlusion.

${ }^{*} P<0.05,{ }^{* *} P<0.001$, as compared with control. 
Table 3. VEGF expression associated with blood vessels

\begin{tabular}{|c|c|c|c|}
\hline Brain region & Control & $24 \mathrm{~h}$ post-UCO & $48 \mathrm{~h}$ post-UCO \\
\hline Corpus callosum & $62.2 \pm 7.2$ & $74.5 \pm 4.6$ & $86.6 \pm 3.4^{*}$ \\
\hline $\begin{array}{l}\text { Subventricular } \\
\text { zone }\end{array}$ & $56.4 \pm 3.4$ & $81.8 \pm 5.4^{*}$ & $82.6 \pm 8.9^{*}$ \\
\hline Stria terminalus & $64.8 \pm 4.7$ & $75.7 \pm 7.6$ & $\begin{array}{c}78.3 \pm 9.2 \\
(n=3)\end{array}$ \\
\hline $\begin{array}{l}\text { Periventricular } \\
\text { white matter }\end{array}$ & $47.2 \pm 4.3$ & $73.3 \pm 7.3^{*}$ & $77.8 \pm 6.0^{*}$ \\
\hline $\begin{array}{l}\text { Subcortical white } \\
\text { matter }\end{array}$ & $45.5 \pm 4.0$ & $72.0 \pm 5.6^{*}$ & $71.2 \pm 5.1^{*}$ \\
\hline Cortex & $50.0 \pm 4.2$ & $68.3 \pm 7.2^{*}$ & $78.9 \pm 2.3^{*}$ \\
\hline $\begin{array}{l}\text { Striatum } \\
\text { (combined) }\end{array}$ & $50.3 \pm 3.1$ & $57.5 \pm 7.5$ & $65.3 \pm 8.4$ \\
\hline Caudate nucleus & $51.8 \pm 6.7$ & $58.0 \pm 12.5$ & $71.2 \pm 10.9$ \\
\hline Putamen & $\begin{array}{c}46.3 \pm 2.7 \\
(n=4)\end{array}$ & $50.5 \pm 3.9$ & $60.6 \pm 13.1$ \\
\hline Internal capsule & $41.5 \pm 3.9$ & $57.4 \pm 7.2$ & $58.9 \pm 8.1$ \\
\hline External capsule & $\begin{array}{c}50.7 \pm 4.0 \\
(n=4)\end{array}$ & $65.5 \pm 6.6$ & $73.3 \pm 7.0$ \\
\hline Claustrum & $56.3 \pm 3.9$ & $55.9 \pm 9.8$ & $62.6 \pm 8.4$ \\
\hline $\begin{array}{l}\text { Thalamus } \\
\text { (combined) }\end{array}$ & $74.1 \pm 1.4$ & $80.5 \pm 7.9$ & $77.0 \pm 6.8$ \\
\hline $\begin{array}{l}\text { Lateral geniculate } \\
\text { nucleus }\end{array}$ & $78.3 \pm 3.1$ & $80.3 \pm 4.3$ & $78.4 \pm 3.8$ \\
\hline $\begin{array}{l}\text { Medial geniculate } \\
\text { nucleus }\end{array}$ & $74.4 \pm 2.7$ & $83.5 \pm 4.3$ & $\begin{array}{c}92.4 \pm 5.4^{*} \\
(n=3)\end{array}$ \\
\hline $\begin{array}{l}\text { Posterior thalamic } \\
\text { nucleus }\end{array}$ & $69.4 \pm 2.9$ & $79.4 \pm 10.2$ & $74.1 \pm 12.3$ \\
\hline Pretectal nucleus & $74.2 \pm 2.9$ & $\begin{array}{c}85.7 \pm 3.5 \\
(n=4)\end{array}$ & $66.1 \pm 9.2$ \\
\hline
\end{tabular}

Percentage of blood vessels immunopositive for VEGF in brain regions of control $(n=5)$, $24 \mathrm{~h}$ post-UCO $(n=5)$, and $48 \mathrm{~h}$ post- $U C O(n=4)$ fetal sheep. Values expressed as mean \pm SEM.

UCO, umbilical cord occlusion; VEGF, vascular endothelial growth factor.

${ }^{*} P<0.05$ as compared with control.

occlusion except, at the $48 \mathrm{~h}$ time point, for the external capsule $\left(455.5 \pm 137.4\right.$ vs. $1,038.8 \pm 169.9$ cells $\left./ \mathrm{mm}^{2}\right)$, the internal capsule $\left(444.8 \pm 137.4\right.$ vs. $\left.1,009.4 \pm 58.2 \mathrm{cells} / \mathrm{mm}^{2}\right)$, and the striatum $\left(516.8 \pm 63.9\right.$ vs. $981.2 \pm 170.4$ cells $\left./ \mathrm{mm}^{2}\right)$. In the other brain regions, the number of VEGF-positive cells ranged from 450 to 900 cells $/ \mathrm{mm}^{2}$, and this density was not changed by UCO; the highest density of VEGF-positive cells was observed in the subventricular zone $\left(1,600-2,000\right.$ cells $\left./ \mathrm{mm}^{2}\right)$, but this was also not changed by UCO.

Cell proliferation, determined from counts of Ki67immunopositive cells, was present throughout the brain of the control fetuses, and ranged from 20 to 100 cells $/ \mathrm{mm}^{2}$ for the majority of brain regions; the highest level of proliferation $\left(\sim 350\right.$ cells $\left./ \mathrm{mm}^{2}\right)$ was observed in the subventricular zone and stria terminalus. A small percentage of blood vessels were associated with Ki67-positive cells (Figure 2), with the highest incidence (5-7\%) occurring in the thalamus. UCO did not affect proliferation in vascular or nonvascular structures

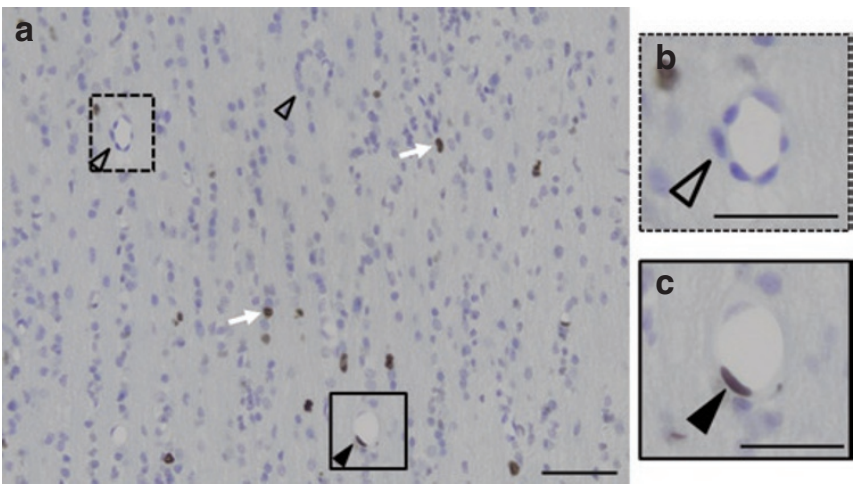

Figure 2. Photomicrograph of Ki67 immunohistochemistry in fetal sheep brain. Ki67-immunopositive staining (white arrow) in the periventricular white matter at $48 \mathrm{~h}$ after UCO. The open arrowheads indicate $(\mathbf{a}, \mathbf{b})$ no Ki67 staining associated with blood vessel, and black arrowhead indicates (c) positive Ki67 staining associated with blood vessel. Taken at $\times 400$ original magnification. (a) Bar $=50 \mu \mathrm{m}$. (b,c) Bar $=25 \mu \mathrm{m}$. UCO, umbilical cord occlusion.
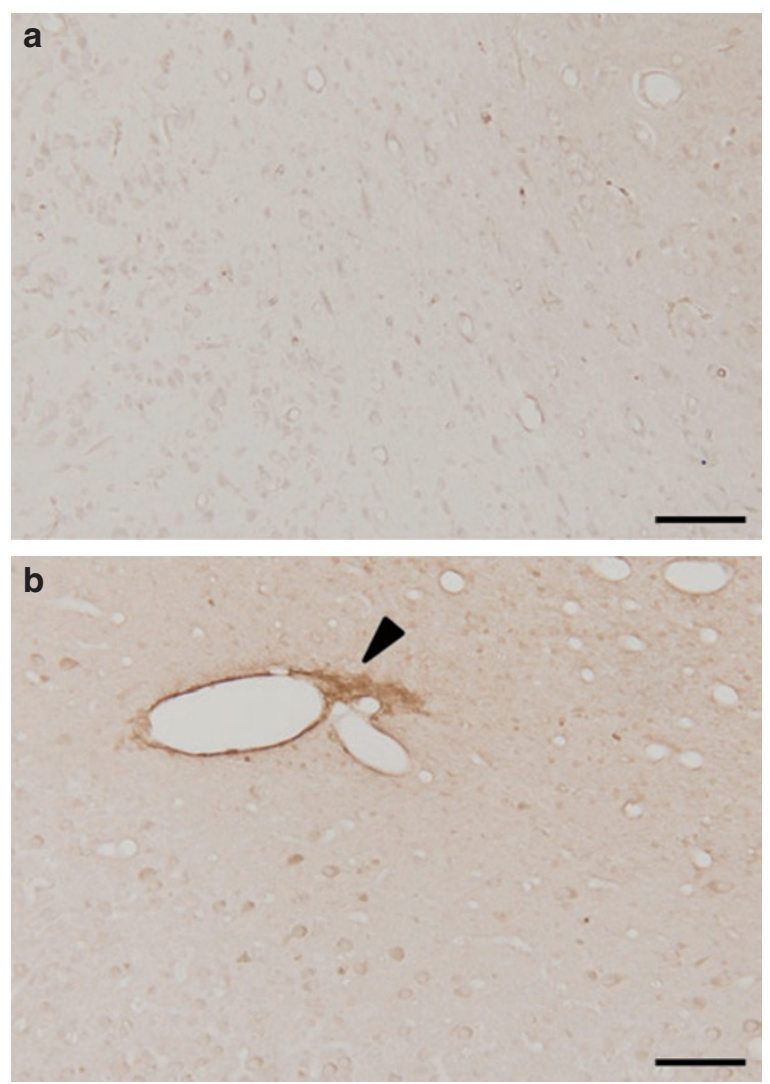

Figure 3. Albumin immunohistochemistry in subcortical white matter of fetal sheep. Albumin immunohistochemistry in subcortical white matter in (a) control and (b) $24 \mathrm{~h}$ post-UCO fetal sheep. Black arrowhead indicates albumin extravasation associated with blood vessel. Taken at $\times 200$ original magnification. Bar $=100 \mu \mathrm{m}$. UCO, umbilical cord occlusion.

except for the subventricular zone, in which the percentage of blood vessels associated with Ki67-positive cells was significantly increased at $24 \mathrm{~h}$ after the UCO $(13.3 \pm 2.1 \%)$ as compared with control $1.7 \pm 0.6 \%$ and $48 \mathrm{~h}$ post-UCO $(4.6 \pm 3.0 \%)$ levels. 
Table 4. Summary of incidence of albumin in fetal sheep brains following UCO

\begin{tabular}{|c|c|c|c|c|c|c|}
\hline \multirow[b]{2}{*}{ Brain region } & \multicolumn{3}{|c|}{ Intracellular expression of albumin } & \multicolumn{3}{|c|}{ Extravasation of albumin } \\
\hline & Control & $24 \mathrm{~h}$ post-UCO & $48 \mathrm{~h}$ post-UCO & Control & $24 \mathrm{~h}$ post-UCO & $48 \mathrm{~h}$ post-UCO \\
\hline Corpus callosum & $5 / 5$ & $5 / 5$ & $3 / 3$ & $0 / 5$ & $0 / 5$ & $0 / 3$ \\
\hline Periventricular white matter & $5 / 5$ & $5 / 5$ & $3 / 3$ & $3 / 5$ & $4 / 5$ & $3 / 3$ \\
\hline Subcortical white matter & $5 / 5$ & $5 / 5$ & $3 / 3$ & $1 / 5$ & $5 / 5$ & $3 / 3$ \\
\hline Subventricular zone & $4 / 5$ & $5 / 5$ & $3 / 3$ & $0 / 5$ & $0 / 5$ & $2 / 3$ \\
\hline Thalamus-LGN & $0 / 5$ & $3 / 5$ & $0 / 4$ & $0 / 5$ & $0 / 5$ & $0 / 4$ \\
\hline Thalamus-PN & $0 / 5$ & $0 / 5$ & $1 / 4$ & $0 / 5$ & $0 / 5$ & $0 / 4$ \\
\hline
\end{tabular}

The incidence of intracellular albumin expression and the occurrence of albumin extravased from blood vessels in control $(n=5)$, and $24 \mathrm{~h}(n=5)$, and $48 \mathrm{~h}(n=3)$ post- $U C \mathrm{C}$ is indicated. Brain regions examined included the corpus callosum, cortex, white matter (periventricular and subcortical), striatum, thalamus, and subventricular zone.

LGN, lateral geniculate nucleus; PN, pretectal nucleus; UCO, umbilical cord occlusion.

Albumin immunoreactivity was identified in the cortex, corpus callosum, and subcortical and periventricular white matter of both control and UCO fetal brains (Figure 3), but clear extravasation of albumin into the brain parenchyma was observed only after UCO (Figure $3 \mathbf{b}$ ). This was particularly evident in periventricular white matter, subcortical white matter, and the subventricular zone at both 24 and $48 \mathrm{~h}$ following UCO (Table 4). No correlation was seen between the percentage of VEGF expression associated with blood vessels and incidence of extravasation at 24 or $48 \mathrm{~h}$ following UCO (data not shown).

\section{DISCUSSION}

VEGF was widely expressed in the late-gestation fetal sheep brain, and we observed strong neuronal expression (particularly in the cortex and striatum), with weaker expression in astrocytes in most regions examined. The main finding of this study was that a severe, global hypoxia caused a significant increase in VEGF expression associated with the vasculature of the subventricular zone, cortex, and some white matter regions in the lategestation fetal sheep brain. In addition, we observed an increase in the incidence of vascular permeability in white matter and the subventricular zone, as shown by extravasation of albumin from blood vessels into the surrounding brain tissue.

The antibody used in this study, raised against purified human VEGF- $\mathrm{A}_{189}$, is likely to recognize binding sites on the folded, tertiary structure of the mature form of the protein. It is possible that other isoforms of VEGF might be present in the fetal sheep brain, but isoforms lacking the full exon 6 domain, which includes all of those commonly found except $\mathrm{VEGF}_{189}$ and $\mathrm{VEGF}_{206}$, are highly diffusible and would probably have been removed by the perfusion of the brain at postmortem. Crossreactivity with $\mathrm{VEGF}_{206}$ is possible, but we are unaware of any data documenting the presence of this isoform in the brain. Therefore, we conclude that the VEGF immunoreactivity observed in the fetal sheep brain arises primarily from $\mathrm{VEGF}_{189}$.

The hypoxic episode produced by interrupting umbilical cord blood flow had little effect on the parenchymal expression of VEGF (except for the striatum, and external and internal capsules). Although neonatal rodent models of hypoxia and stroke have found increased VEGF following hypoxia, in these studies exposure to hypoxia was either more prolonged $(1.5 \mathrm{~h}$ middle cerebral artery occlusion) (11); $2 \mathrm{~h}$ hypobaric hypoxia (12), or chronic (30 d low inspired oxygen) (14). In the current study, hypoxia resulted in increased expression of VEGF associated with blood vessels in the subventricular zone, periventricular and subcortical white matter, and the corpus callosum. The cortex (examined at the level of the motor and somatosensory cortexes) and the thalamic medial geniculate nucleus were the only neuron-rich areas in which vascular-associated VEGF was significantly increased. Of note, we did not observe any cellular changes indicating apoptosis within or around blood vessels in which increased VEGF expression occurred.

VEGF is a mitogen considered important for endothelial cell survival and activation; its increased expression leads to angiogenesis and vascular remodeling $(26,27)$. Increased VEGF has also been seen in the neovascular sprouts in the necrotic foci in brains of infants with periventricular leukomalacia (8). However, a VEGF-driven mitogenic response following hypoxia in the fetal sheep brain seems unlikely because we saw no effect on cell proliferation in general, and increased Ki67 immunoreactivity associated with blood vessels was observed only in the subventricular zone. Proliferation associated with blood vessels might occur after the $48 \mathrm{~h}$ survival time used in this study, as has been seen in the adult rat brain following hypoxia (20).

The increased incidence of extravasation of serum albumin, observed predominantly in periventricular and subcortical white matter and the subventricular zone, is consistent with alterations in vascular permeability, although there appears to be little direct spatial correlation between the increased VEGF expression associated with blood vessels and the regions of increased vascular leakage of albumin. Extravasation did not occur in all brain regions that showed increased VEGF expression following UCO, suggesting other mechanisms may contribute to the increased vascular permeability. To fully understand the role of the increased expression of VEGF in the fetal brain, it may be necessary to block the actions of VEGF using a blocking peptide, as shown for the adult rat in which such treatment prevented hypoxia-induced vascular leakage (16). 
In vitro studies have shown VEGF-mediated reduction in expression of the tight junction proteins occludin and zona occludin-1 $(28,29)$, which may be a primary reason for the increased permeability of the blood-brain barrier when VEGF expression is increased. However, other contributory factors could include increased expression of the aquaporin 4 water channel in astrocytes (30), and downregulation of angiopoietin-1, an important accessory protein for neovascularization and producing stable blood vessel structure, which is also correlated with increased blood-brain barrier leakage (31).

In addition to the presence of albumin outside vascular structures, we also noted the intracellular albumin in many regions of the control and UCO fetal sheep brains, confirming previous observations from the cerebellum of fetal sheep (32). The functional significance of the cellular uptake of albumin in the brain is still under debate, but increased albumin in astrocytes, activated microglia, or endothelial cells may be associated with phagocytosis, whereas both protective and toxic effects of albumin uptake by neurons have also been described (33).

The method used to quantify the presence of extravasation, although crude, is more accurate than counting the number of blood vessels because protein leak may occur from one vessel and it is difficult to identify which one accurately. Measuring the area of extravasation can also be inaccurate because it is dependent on the plane in which sections are cut. A limitation of this study is that we were unable to find an accurate marker for endothelial cells and pericytes for the fetal sheep brain. Pericytes are intimately associated with the basement membrane and communicate with endothelial cells, and they have been suggested to be cells that, by increasing VEGF expression, respond earliest to hypoxia and mediate opening of the blood-brain barrier (34-36).

Notwithstanding this, our results show that the microvasculature, particularly in white matter regions known to be sites of injury in the human neonate (37), respond to $10 \mathrm{~min}$ of severe global hypoxia with upregulation of VEGF expression and increased leakage of plasma protein. Although further studies are required to investigate the complex nature of VEGF expression in the neonatal brain, the findings from this study suggest that in a relatively short-term period (i.e., up to $48 \mathrm{~h}$ following UCO), the response of the microvasculture could aid in understanding the regional differences in vulnerability to injury of the term brain.

\section{METHODS}

\section{Animal Surgery and Experimentation}

Under prior approval from the School of Biomedical Science Animal Ethics Committee of Monash University, 14 pregnant Border-Leicester ewes carrying singleton fetuses were used in this study. As previously described $(24,25)$, surgery was performed under general anesthesia at $124-126 \mathrm{~d}$ gestational age (term is 146 days), induced by an intravenous injection of $1 \mathrm{~g}$ sodium thiopentone $(50 \mathrm{mg} / \mathrm{ml}$; Pentothal, Boehringer Ingelheim Pty, North Ryde, Australia) in $20 \mathrm{ml}$ sterile water, with anesthesia being then maintained by inhalation of $1.5-2 \%$ isoflurane (Isoflo, Abbott Australasia Pty, Kurnell, Australia). A polyvinyl catheter filled with heparinized saline was inserted into a fetal femoral artery to obtain blood samples and measure arterial pressure and heart rate. A second saline-filled catheter was placed in the amniotic sac for a pressure reference. An inflatable silastic cuff (type OC-16; In Vivo Metric, Healdsburg, CA) was placed around the abdominal end of the umbilical cord, which on inflation was known to be able to result in complete occlusion of the umbilical arteries and veins. The fetus was then returned to the uterus and the incision was closed. All catheters were exteriorized through a maternal flank incision, and the maternal abdominal incision was closed.

Experiments were performed 4-5 d after surgery. Fetal arterial and amniotic pressure were measured using solid-state pressure transducers, and fetal arterial pressure was calculated by electronic subtraction of amniotic pressure. Fetal heart rate was calculated online from the blood pressure pulse. All data were recorded continuously on the hard disk of a computer via an analog-digital converter and Chart software (PowerLab, ADInstruments, Bella Vista, Australia). On the day of the experiment, $\mathrm{UCO}$ was done for $10 \mathrm{~min}$ in nine fetuses by inflating the cuff with $\sim 3 \mathrm{ml}$ of sterile water; in the other five fetuses, a sham cuff inflation procedure was performed. Fetal arterial blood samples $(0.5 \mathrm{ml})$ were taken at $-1 \mathrm{~h},-5 \mathrm{~min},+5 \mathrm{~min},+9 \mathrm{~min}, 30 \mathrm{~min}$, and $1,2,4,6,8$, 10,12 , and $24 \mathrm{~h}$ relative to the start of the occlusion (time $=0$ ). Blood samples were used immediately to measure $\mathrm{pO}_{2}, \mathrm{pCO}_{2}, \mathrm{O}_{2}$ saturation, $\mathrm{pH}$, and hematocrit using a Radiometer ABL5 analyser (Radiometer Medical ApS, Brønshøj, Denmark), and blood glucose and lactate concentrations were measured using a YSI 2300 STAT Glucose and Lactate Analyser (YSI Life Sciences, Yellow Springs, OH). At $24 \mathrm{~h}(n=5)$ or $48 \mathrm{~h}(n=4)$ after the actual or sham UCO $(n=5 ; 24 \mathrm{~h})$, the ewe and fetus were humanely killed by intravenous injection of pentobarbitone sodium (Lethabarb, Virbac Pty, Regents Park, Australia) to the ewe. The fetal brain was transcardially perfused with 11 of sterile saline and then with $4 \%$ paraformaldehyde before being removed from the skull.

\section{Immunohistochemistry}

Single-label immunohistochemistry. Immunohistochemistry was performed on $10 \mu \mathrm{m}$ paraffin-embedded sections at two anatomical levels of the brain (section 720: subventricular zone, stria terminalus, corpus callosum, striatal regions (caudate nucleus, putamen, claustrum, internal and external capsule, cortex (between the motor and somatosensory cortexes)), and periventricular and subcortical white matter, and section 1080: thalamus (lateral geniculate, medial geniculate, posterior thalamic, and pretectal nuclei), Sheep Ovis Aries atlas; https://www.msu.edu/ brains/brains/sheep/index. html. All sections were placed on Superfrost plus glass slides (Mezel Glaser, Braunschweig, Germany), dewaxed and rehydrated through serial ethanols (100 and 70\%). Sections were incubated overnight at $4^{\circ} \mathrm{C}$ with (i) VEGF mouse monoclonal antibody (VEGF- ${ }_{189}, 1: 300$; Novus Biologicas, Littleton, CO) made up in True Vision (Sapphire Bioscience, Waterloo, Australia); (ii) Ki67 rabbit monoclonal antibody (1:100, Thermo Fisher Scientific, Kalamazoo, MI) made up in $0.1 \mathrm{~mol} / \mathrm{l}$ phosphate buffered saline; or (iii) sheep antialbumin (1:1,000, Accurate Chemical \& Scientific Corporation, Westbury, NY) made up in DAKO Real Antibody Diluent (DAKO Australia, Campbellfield, Australia). Antigen retrieval was carried out as follows: (i) VEGF, $0.05 \mathrm{~mol} / \mathrm{l}$ tris buffered saline $(\mathrm{pH} \mathrm{10})$ for $3 \times 10 \mathrm{~min}$ in a microwave oven and cooled for $30 \mathrm{~min}$; (ii) Ki67, $0.01 \mathrm{~mol} / \mathrm{l}$ citric acid buffer ( $\mathrm{pH}$ 6) in a microwave oven $(3 \times 10 \mathrm{~min})$; and (iii) albumin, no antigen retrieval. Sections were then incubated in $0.3 \%$ hydrogen peroxide $\left(\mathrm{H}_{2} \mathrm{O}_{2}\right)$ for $10 \mathrm{~min}$ at room temperature and the appropriate blocking solution ( $2 \%$ normal rabbit serum, for VEGF; $5 \%$ normal goat serum and $2 \%$ bovine serum albumin for Ki67; DAKO protein free block for albumin) for $45 \mathrm{~min}$ at room temperature. The sections were then incubated with the appropriate biotinylated secondary antibody (goat antirabbit, 1:200, Vector Laboratories, Burlingame, CA or rabbit antimouse, 1:200, DAKO Australia) for $1 \mathrm{~h}$, washed with phosphate buffered saline, followed by $1 \mathrm{~h}$ incubation with streptavidin horseradish peroxidase (1:200 Amersham Bioscience, Castle Hill, Australia). Staining was then visualized using metal-enhanced diaminobenzidine (Pierce Biotechnology, Rockford, IL). A negative control slide was always included in each run by omitting the primary antibody from the incubation buffer; in all cases, no staining was present.

Double-label immunohistochemistry. Sections were incubated with either monoclonal antiglial fibrillary acidic protein (1:100; Sigma, 
St Louis, MO) or antimicrotubule-associated protein 2 (1:200; NeoMarkers, Fremont, CA) to identify astrocytes and neurons, respectively, and mouse monoclonal antibody to identify VEGF (1:200). Immunoreactivity was visualized with Alexa Fluor 488 goat antimouse (1:1,000; Invitrogen Life Technologies Australia, Mulgrave, Australia) for glial fibrillary acidic protein or microtubule-associated protein 2 and Alexa Fluor 594 goat antimouse (1:1,000; Invitrogen Life Technologies Australia, Mulgrave, Australia) for VEGF.

Quantification of immunoreactivity. All slides were coded, with counting and other assessments done by an examiner blinded to the source of the material. Images were taken under light microscopy ( $\times 400$ magnification; Olympus BX41; DP25 Camera, Olympus Australia, Mt. Waverly, Australia). The numbers of Ki67 and VEGF-immunopositive cells were counted using Image J (v1.6.0 for Windows; National Institutes of Health, Bethesda, MD). For each region, the results of three fields of view per section for two adjacent (duplicate) sections were averaged for each animal, and then the results averaged for all animals in each group: control $(n=5), 24 \mathrm{~h}$ post-UCO $(n=5)$, and $48 \mathrm{~h}$ post-UCO $(n=4)$. The numbers of blood vessels expressing either Ki67 or VEGF were counted and expressed as a percentage of the total number of blood vessels per field of view. Double-label fluorescent images were visualized with fluorescence (red and green filters) microscopy using an Olympus BX41 (Olympus Australia). Albumin immunohistochemistry was assessed for the extravasation of albumin as previously described $(32,38)$. Images were taken at $\times 200$ magnification.

\section{Data Analysis}

All data are shown as mean \pm SEM. A Shapiro-Wilks test for normality was conducted, and if passed $(P>0.05)$, a parametric test was then run. A two-way mixed-model ANOVA for time (within subjects) and treatment (between subjects) was used to analyze the blood gases, $\mathrm{pH}$, blood pressure, and heart rate, using a Bonferroni post hoc test. Cell counts in each brain region of the sham control, $24 \mathrm{~h}$ post-UCO, and $48 \mathrm{~h}$ post-UCO groups were compared using a one-way ANOVA, with a Student-Neuman-Keul post hoc test applied, if required. If data were not normally distributed, transformations ( $\log _{10}$, exponential, and square root) were conducted, and if these failed to achieve normality a nonparametric Kruskal-Wallis test was conducted with pairwise comparisons. All statistical tests were done using SPSS v18 (SPSS, Chicago, IL) for Windows, with significance set at $P<0.05$.

\section{ACKNOWLEDGMENTS}

We thank Alex Satragno and Edwin Yan for their help with all the fetal sheep surgeries and Liliana Jackanic and staff in the Department of Physiology animal house for assistance with the postsurgical care of the animals.

\section{STATEMENT OF FINANCIAL SUPPORT}

This study was supported by grants from the National Health and Medical Research Council Australia and Cerebral Palsy Alliance (to M.C.-M. and D.W.W.) and to the Monash Institute of Medical Research from the Victorian Infrastructure Support Fund.

\section{REFERENCES}

1. Cowan F, Rutherford M, Groenendaal F, et al. Origin and timing of brain lesions in term infants with neonatal encephalopathy. Lancet 2003;361:736-42.

2. McLean C, Ferriero D. Mechanisms of hypoxic-ischemic injury in the term infant. Semin Perinatol 2004;28:425-32.

3. Mallard EC, Williams CE, Johnston BM, Gluckman PD. Increased vulnerability to neuronal damage after umbilical cord occlusion in fetal sheep with advancing gestation. Am J Obstet Gynecol 1994;170(1 Pt 1): 206-14.

4. Van den Broeck C, Himpens E, Vanhaesebrouck P, Calders P, Oostra A. Influence of gestational age on the type of brain injury and neuromotor outcome in high-risk neonates. Eur J Pediatr 2008;167:1005-9.

5. Aly H, Hassanein S, Nada A, Mohamed MH, Atef SH, Atiea W. Vascular endothelial growth factor in neonates with perinatal asphyxia. Brain Dev 2009;31:600-4.
6. Vasiljevic B, Maglajlic-Djukic S, Gojnic M, Stankovic S, Ignjatovic S, Lutovac D. New insights into the pathogenesis of perinatal hypoxicischemic brain injury. Pediatr Int 2011;53:454-62.

7. Trollmann R, Amann K, Schoof E, et al. Hypoxia activates the human placental vascular endothelial growth factor system in vitro and in vivo: up-regulation of vascular endothelial growth factor in clinically relevant hypoxic ischemia in birth asphyxia. Am J Obstet Gynecol 2003;188:517-23.

8. Arai Y, Deguchi K, Takashima S. Vascular endothelial growth factor in brains with periventricular leukomalacia. Pediatr Neurol 1998; 19:45-9.

9. Sentilhes L, Michel C, Lecourtois M, et al. Vascular endothelial growth factor and its high-affinity receptor (VEGFR-2) are highly expressed in the human forebrain and cerebellum during development. J Neuropathol Exp Neurol 2010;69:111-28.

10. Ogunshola OO, Stewart WB, Mihalcik V, Solli T, Madri JA, Ment LR. Neuronal VEGF expression correlates with angiogenesis in postnatal developing rat brain. Brain Res Dev Brain Res 2000;119:139-53.

11. Mu D, Jiang X, Sheldon RA, et al. Regulation of hypoxia-inducible factor 1alpha and induction of vascular endothelial growth factor in a rat neonatal stroke model. Neurobiol Dis 2003;14:524-34.

12. Kaur C, Sivakumar V, Ang LS, Sundaresan A. Hypoxic damage to the periventricular white matter in neonatal brain: role of vascular endothelial growth factor, nitric oxide and excitotoxicity. J Neurochem 2006; 98:1200-16.

13. Breier G, Albrecht U, Sterrer S, Risau W. Expression of vascular endothelial growth factor during embryonic angiogenesis and endothelial cell differentiation. Development 1992;114:521-32.

14. Ment LR, Stewart WB, Fronc R, et al. Vascular endothelial growth factor mediates reactive angiogenesis in the postnatal developing brain. Brain Res Dev Brain Res 1997;100:52-61.

15. Zhang ZG, Zhang L, Jiang Q, et al. VEGF enhances angiogenesis and promotes blood-brain barrier leakage in the ischemic brain. J Clin Invest 2000;106:829-38.

16. Schoch HJ, Fischer S, Marti HH. Hypoxia-induced vascular endothelial growth factor expression causes vascular leakage in the brain. Brain 2002;125(Pt 11):2549-57.

17. Dvorak HF, Brown LF, Detmar M, Dvorak AM. Vascular permeability factor/vascular endothelial growth factor, microvascular hyperpermeability, and angiogenesis. Am J Pathol 1995;146:1029-39.

18. Kim KJ, Li B, Winer J, et al. Inhibition of vascular endothelial growth factor-induced angiogenesis suppresses tumour growth in vivo. Nature 1993;362:841-4.

19. Bates DO, Jones RO. The role of vascular endothelial growth factor in wound healing. Int J Low Extrem Wounds 2003;2:107-20.

20. Marti HJ, Bernaudin M, Bellail A, et al. Hypoxia-induced vascular endothelial growth factor expression precedes neovascularization after cerebral ischemia. Am J Pathol 2000;156:965-76.

21. McIntosh GH, Baghurst KI, Potter BJ, Hetzel BS. Foetal brain development in the sheep. Neuropathol Appl Neurobiol 1979;5:103-14.

22. Mallard EC, Gunn AJ, Williams CE, Johnston BM, Gluckman PD. Transient umbilical cord occlusion causes hippocampal damage in the fetal sheep. Am J Obstet Gynecol 1992;167:1423-30.

23. Miller SL, Yan EB, Castillo-Meléndez M, Jenkin G, Walker DW. Melatonin provides neuroprotection in the late-gestation fetal sheep brain in response to umbilical cord occlusion. Dev Neurosci 2005;27:200-10.

24. Castillo-Meléndez M, Yan E, Walker DW. Expression of erythropoietin and its receptor in the brain of late-gestation fetal sheep, and responses to asphyxia caused by umbilical cord occlusion. Dev Neurosci 2005;27:220-7.

25. Yan EB, Baburamani AA, Walker AM, Walker DW. Changes in cerebral blood flow, cerebral metabolites, and breathing movements in the sheep fetus following asphyxia produced by occlusion of the umbilical cord. Am J Physiol Regul Integr Comp Physiol 2009;297:R60-9.

26. Breier G. Functions of the VEGF/VEGF receptor system in the vascular system. Semin Thromb Hemost 2000;26:553-9.

27. Leung DW, Cachianes G, Kuang WJ, Goeddel DV, Ferrara N. Vascular endothelial growth factor is a secreted angiogenic mitogen. Science 1989;246: $1306-9$. 
28. Wang W, Dentler WL, Borchardt RT. VEGF increases BMEC monolayer permeability by affecting occludin expression and tight junction assembly. Am J Physiol Heart Circ Physiol 2001;280:H434-40.

29. Fischer S, Wobben M, Marti HH, Renz D, Schaper W. Hypoxia-induced hyperpermeability in brain microvessel endothelial cells involves VEGFmediated changes in the expression of zonula occludens- 1 . Microvasc Res 2002;63:70-80.

30. Kaur C, Sivakumar V, Zhang Y, Ling EA. Hypoxia-induced astrocytic reaction and increased vascular permeability in the rat cerebellum. Glia 2006;54:826-39.

31. Zhang ZG, Zhang L, Tsang W, et al. Correlation of VEGF and angiopoietin expression with disruption of blood-brain barrier and angiogenesis after focal cerebral ischemia. J Cereb Blood Flow Metab 2002;22: 379-92.

32. Hutton LC, Castillo-Melendez M, Walker DW. Uteroplacental inflammation results in blood brain barrier breakdown, increased activated caspase 3 and lipid peroxidation in the late gestation ovine fetal cerebellum. Dev Neurosci 2007;29:341-54.
33. Alonso A, Reinz E, Fatar M, Hennerici MG, Meairs S. Clearance of albumin following ultrasound-induced blood-brain barrier opening is mediated by glial but not neuronal cells. Brain Res 2011;1411:9-16.

34. Yamagishi S, Yonekura H, Yamamoto Y, et al. Vascular endothelial growth factor acts as a pericyte mitogen under hypoxic conditions. Lab Invest 1999;79:501-9.

35. Gonul E, Duz B, Kahraman S, Kayali H, Kubar A, Timurkaynak E. Early pericyte response to brain hypoxia in cats: an ultrastructural study. Microvasc Res 2002;64:116-9.

36. Al Ahmad A, Taboada CB, Gassmann M, Ogunshola OO. Astrocytes and pericytes differentially modulate blood-brain barrier characteristics during development and hypoxic insult. J Cereb Blood Flow Metab 2011;31:693-705.

37. Iwata S, Bainbridge A, Nakamura T, et al. Subtle white matter injury is common in term-born infants with a wide range of risks. Int J Dev Neurosci 2010;28:573-80.

38. Polglase GR, Nitsos I, Baburamani AA, et al. Inflammation in utero exacerbates ventilation-induced brain injury in preterm lambs. J Appl Physiol 2012;112:481-9. 